\title{
Immunological factors and risk of infection in plateau phase myeloma
}

\author{
R M Hargreaves, J R Lea, H Griffiths, J A Faux, J M Holt, C Reid, C Bunch, M Lee, \\ H M Chapel
} Corporation, Hyland Division, Glendale, August 1994

Most patients with myeloma have an acquired polyclonal hypogammaglobulinaemia, the aetiology of which remains obscure. The precise significance and nature of this antibody deficiency in relation to infection is not defined although extrapolation from primary hypogammaglobulinaemia, where the risk of bacterial infection at almost any site is considerably increased, suggests a direct correlation. Impaired cell mediated immunity is also important in susceptibility to fungal and viral infections, but these are relatively uncommon in myeloma patients ${ }^{1}$ and so $\mathrm{T}$ cell defects may be less clinically significant. There is no clear correlation between immunosuppression and tumour mass; the contribution of chemotherapy to infection, particularly through neutropenia, is also unclear. ${ }^{2}$ Immunisation responses are also impaired, ${ }^{3}$ although no correlation with infection has been demonstrated.

Infection has been recognised as a frequent and serious complication in patients with myeloma since the original description of the disease. Studies over the past 40 years have demonstrated a high but variable incidence of bacterial infection. ${ }^{1-4}$ In 1973 Twomey and Houston reported a 15-fold increase in the incidence of infection in patients with myeloma compared with an aged matched control group. ${ }^{1}$ They found that infecting organisms were predominantly Gram positive bacteria, but in recent years the pattern of infecting organisms has altered to include more Gram negative infections. ${ }^{5}$

Chemotherapy regimes for myeloma have varied in both intensity and duration between studies, and their contribution to infection has proved difficult to estimate. No studies have looked at possible predictors of infection prospectively, though prognostic factors for survival have been studied extensively. ${ }^{6}$

This prospective study was undertaken to determine the present rate and pattern of infection in patients with plateau phase myeloma in the United Kingdom and to identify immunological parameters predictive of infection risk. Attempts were made to define subgroups of patients at particular risk by investigating associations between infection and humoral immunity (including specific antibody titres and IgG subclass levels), neutrophil counts, and immunisation responses (in a subgroup of subjects). In addition, age, sex, paraprotein type, disease stage characteristics (stage, performance status, presence of urinary free light chains, haemoglobin and creatinine concentrations), and treatment with chemotherapy
Keywords: Plateau phase myeloma, risk factors, infection.

\begin{abstract}
(f Clin Pathol 1995;48:260-266) genous antigens, who are at increased risk may benefit from, can be identified globulin therapy to reduce the risk of in$(0.49)$. The predominant site of infection
was the respiratory tract. Clinical and laboratory parameters showed only IgA levels to be significantly associated with at least one serious infection. Conclusions-A subgroup of patients with genel antigens, nificantly reduced and a poor IgG response
to Pneumovax II immunisation was associated with an increased risk of septicaemia and low IgG2 levels. The overall serious infection rate was 0.92 infections
per patient year and was four times higher during periods of active disease (1.90) compared with plateau phase myeloma was the respiratory tract. Clinical and sex and reduced non-paraprotein IgG and fection.
\end{abstract}$$
\text { centrations), and treatment with chemotherapy }
$$ 
Table 1 Classification of infection by severity

\begin{tabular}{ll}
\hline Major* & Septicaemia (culture positive) \\
& PUO (fever $\geqslant 39 \cdot 5^{\circ} \mathrm{C}$ : culture negative) \\
& Meningitis \\
& Pneumonia (with $x$ ray evidence) \\
& URTI complicated by secondary bacterial infection \\
& Acute bronchitis (not requiring hospital admission) \\
& Pleurisy \\
& UTI (culture positive or $>100$ pus cells/hpf) \\
& PUO (fever $\geqslant 38 \cdot 5^{\circ} \mathrm{C}$ but $<39 \cdot 5^{\circ} \mathrm{C}$ : culture negative) \\
& Skin abscess/cellulitis \\
& Localised herpes zoster \\
& Uncomplicated URTI \\
& Minor skin infection \\
Minor* & UTI (30-100 pus cells/hpf: culture negative) \\
\hline
\end{tabular}

* Serious infection $=$ major + moderate infections; PUO = pyrexia of unknown origin; URTI = upper respiratory tract infection; UTI = urinary tract infection; $h p f=h i g h$ power field.

were examined to determine whether any correlation with infection could be shown.

\section{Methods}

The criteria used for the definition of myeloma were those of the Medical Research Council. ${ }^{7}$ All patients attending routine outpatient clinics in Oxford and Harrow were invited to enter the study; no patient refused. From March 1987 to September 1989,102 patients with myeloma were recruited. Of these patients, 84 had established disease (in different phases). The average time from diagnosis was 30.5 months (median 23 months) with 19 long term survivors (more than 48 months). Eighteen new patients were entered as soon as the diagnosis was confirmed. Patients were monitored for infections for a period of between one and 25 months before consideration for immunisation. A subgroup of 41 patients selected by disease stability (to permit adequate follow up) and willingness to participate in further investigations, were subsequently immunised with Pneumovax II and tetanus and diphtheria toxoids. Following immunisation, patients were removed from the infection monitoring part of the study. A control population of healthy elderly individuals with a similar age and sex distribution to the patients (62 volunteers, aged 55-90 years) was used for comparison of spontaneous specific antibody titres, total immunoglobulin and IgG subclass levels. Ethical Committee approval was obtained from the local ethical committees for the monitoring of infections and detailed immunological assessment including immunisation, and for the collection of specimens from the control population. All patients were required to sign a consent form on entry to the study, and those subsequently immunised completed another consent form before immunisation.

INFECTION MONITORING AND CLASSIFICATION Details of diagnosis, treatment and history of past infection since diagnosis were obtained on entry to the study from the patient and the hospital records. Patients kept a daily diary of any symptoms suggestive of infection. They were followed every four weeks in the outpatient clinic when diary charts were reviewed and details of disease symptoms, chemotherapy and any infection, however trivial, were recorded. At this time, blood samples were taken for full blood count (including neutrophil count), routine biochemistry and immunoglobulin, paraprotein, and beta-2-microglobulin measurements. Immunosuppression was defined as non-paraprotein immunoglobulin concentrations below the laboratory lower limit of normal (IgG $\leq 6 \mathrm{~g} / \mathrm{dl} ; \operatorname{IgA} \leq$ $0.8 \mathrm{~g} / \mathrm{dl} ; \operatorname{IgM} \leq 0.4 \mathrm{~g} / \mathrm{dl})$. All patients admitted to hospital with an infection were visited and full details of the episode, including neutrophil count, C-reactive protein concentration and any microbiological culture recorded.

Each infection was classified as bacterial, viral, fungal or unknown, on consideration of clinical, radiological and laboratory findings. The latter included relevant microbiological culture, polymorph neutrophil leucocytosis (greater than $90 \%$ neutrophils on white cell count differential) and raised C-reactive protein concentration (greater than $5 \mathrm{mg} / \mathrm{dl}$ ). Infections were divided into major, moderate or minor; serious infections comprised all major and moderate infections (table 1).

\section{IMMUNOLOGICAL INVESTIGATIONS}

Serum was collected on entry to the study for measurement of specific IgG antibody titres to four extrinsic antigens: tetanus and diphtheria toxoids (toxoid vaccines used as antigen), pneumococcal capsular polysaccharides (using Pneumovax II vaccine (MSD) containing 23 capsular types as antigens) and Escherichia coli lipopolysaccharide (LPS) (E coli 026.B6 LPS (Sigma) as antigen). In the 41 patients who were immunised specific IgG antibody titres to tetanus and diphtheria toxoids and Pneumovax II polysaccharides were also measured before and four weeks after immunisation. All specific antibody titres were measured by enzyme linked immunosorbent assay (ELISA): the method used for tetanus, diphtheria and $E$ coli antibody measurements was identical with that previously described for antipneumococcal antibody measurement ${ }^{8}$ using the antigens outlined above. A National Institute for Biological Standards standard was used for tetanus (hence measurements presented as international units (IU)). No standards were available for the other antibodies and thus arbitrary scales in "units" were established. IgG subclasses were measured by radial immune diffusion (Binding Site monoclonal IgG subclass RID plates (IgG1, 2, 3 and 4)).

\section{DEFINITION OF IMMUNISATION RESPONSES}

Immunisation responses were classified as poor, intermediate or good on the basis of the difference between the pre- and postimmunisation IgG antibody titres. Good responders showed an increase of at least twofold as well as a postimmunisation titre reaching a preset minimum value based on the normal range for age calculated from the baseline results obtained in the control population. Poor 
responders showed no difference between preand postimmunisation titres; intermediate responders failed to produce an adequate enough response to be included in the good response group.

\section{INFECTION TIMING AND PREDICTORS}

Disease progression was divided into four phases, based on the Medical Research Council definition of plateau phase in myeloma. ${ }^{9}$ All newly diagnosed patients were designated as being in induction phase. Plateau phase was defined as minimal disease symptoms or asymptomatic patients with stable paraprotein and beta-2-microglobulin measurements over three months. Patients neither in induction nor plateau phase were defined as having either relapsed disease if receiving chemotherapy for recurrence of symptoms or progressive disease.

Infection rates were calculated by dividing the recorded number of infections by the length of the observation period (in patient years); this was done for all infections and serious (major and moderate) infections. Episodes of infection were grouped by month of occurrence to ascertain seasonal variation. Disease phase was noted at the time of each serious infection to investigate whether there was clustering of infection in a particular phase and infection rates were calculated separately for induction, plateau and relapsed/progressive disease phases.

To study possible predictors of serious infection, patients were divided into two groups: those in whom serious (major and/or moderate) infections were recorded and those with only minor infections or none at all during the study period. In addition to the immunological parameters already described, other patient and disease characteristics were also compared between these two groups; these included age, sex, paraprotein type, disease stage, performance status, the presence of free urinary light chains, and type of chemotherapy regime received, if any, at the time of the infection. Absolute neutrophil counts were also examined in relation to disease phase, chemotherapy and occurrence of serious infection. Infections recorded retrospectively (before the start of the study) were included in the analysis only where there was good documentary evidence of infection; their inclusion has been specified in the results.

The extent of disease was staged as described by Durie and Salmon. ${ }^{10}$ Performance status was assessed at diagnosis and on entry to the study, employing the four categories used in current Medical Research Council Myeloma Trials, namely asymptomatic, minimal disease symptoms, restricted activity due to disease, and bedbound. ${ }^{11}$

\section{CHEMOTHERAPY REGIMES}

These were divided into four groups: none, oral alkylating agents only (for example, intermittent pulsed melphalan in conventional dosage $\left.\left(7 \mathrm{mg} / \mathrm{m}^{2}\right)\right)$, combined oral/intravenous conventional dose combination regimes (including ABCM (Adriamycin, BCNU, cyclophosphamide, melphalan) and VCMP/ VBAP (vincristine, cyclophosphamide, melphalan, prednisolone/vincristine, BCNU, Adriamycin, prednisolone)) and intensive conditioning regimes (including VAD (vincristine, Adriamycin, dexamethasone) or VAMP (vincristine, Adriamycin, methylprednisolone) and high dose melphalan $\left.\left(140 \mathrm{mg} / \mathrm{m}^{2}\right)\right)$.

\section{STATISTICS}

All qualitative data (for example, proportion of infections in different seasons of the year) were analysed by the usual $\chi^{2}$ test; if only two categories were involved, a correction for continuity was used. Quantitative data were compared between the two groups of patients (with and without serious infections) by the two-sample $t$ test using a logarithmic transformation to achieve normality as required.

Stepwise regression analysis was used to identify a set of predictors for the presence of serious infection. Logistic regression analysis was employed to establish the actual regression equation relating infection to this prediction set. $^{12}$

\section{Results}

The patients $(n=102)$ were observed prospectively for a total of 84.8 patient years; 78 episodes of serious infection occurred in 49 patients during this period. The mean observation period for each patient was 10 months with a range of one to 25 months. The overall serious infection rate was 0.92 infections per patient year including infection related deaths (table 2).

\section{SPECIFIC ANTIBODY LEVELS}

Levels of specific IgG antibodies were significantly lower in patients with myeloma compared with the control population for

Table 2 Infections by severity, site and type

\begin{tabular}{lc}
\hline Infection class & No. of infections \\
\hline Minor & 94 \\
& 45 \\
Moderate & 19 \\
respiratory tract (B) & 10 \\
urinary tract (B) & 2 \\
PUO (U) & 2 \\
skin (B) & 6 \\
herpes zoster (V) & 6 \\
other (U) & 22 \\
Major & 5 \\
septicaemia (B) & 3 \\
PUO/other (U) & 0 \\
meningitis (B) & 14 \\
pneumonia (B) & 11 \\
Resulting in death & 5 \\
septicaemia (b) & 6 \\
pneumonia (b) & 78 \\
Total serious infections & 0.92 \\
Serious infection rate* &
\end{tabular}

Infection type: $\mathrm{B}=$ bacterial; $\mathrm{U}=$ unknown; $\mathrm{V}=$ viral; $\mathrm{PUO}=$ pyrexia of unknown origin. ${ }^{*}$ Major and moderate infections per patient year. 
Table 3 Spontaneous specific IgG antibody titres (results represented as median (range))

\begin{tabular}{|c|c|c|c|c|}
\hline Patient group & $\begin{array}{l}\text { Pneumococcal } \\
\text { polysaccharides }(\mathrm{U} / \mathrm{ml})\end{array}$ & $E$ coli LPS (U/ml) & Tetanus (IU/ml) & Diphtheria (U/ml) \\
\hline $\begin{array}{l}\text { Controls }(n=62) \\
\text { Myeloma }\end{array}$ & $63(0-241)$ & $11(0-100)$ & $0.40(0-1 \cdot 3)$ & $8(1-122)$ \\
\hline $\begin{array}{l}\text { all }(n=102) \\
\text { serious infections }(n=49) \\
\text { no serious infections }(n=53)\end{array}$ & $\begin{array}{l}12(0-241) \\
12 \\
11\end{array}$ & $\begin{array}{l}5(0-93) \\
5 \\
5\end{array}$ & $\begin{array}{l}0.05(0-1 \cdot 3) \\
0.06 \\
0.04\end{array}$ & $\begin{array}{l}2(0-72) \\
3 \\
2\end{array}$ \\
\hline
\end{tabular}

antitetanus $(p<0.0001)$, antidiphtheria $(p=$ $0.0022)$ and antipneumococcal antibodies $(\mathrm{p}<0.0001)$. Anti-E coli LPS antibodies were reduced but the difference was not statistically significant (table 3 ). Fifteen patients had low levels $(<2$ SD below the mean for controls (on a log scale) of both antipneumococcal and anti$E$ coli spontaneous antibody titres and at least one serious infection since diagnosis; this compared with only two patients with such low titres who had no serious infections $(p=0.035)$. Fifty nine patients had low levels $(<1$ SD below mean for controls (on a log scale) of antipneumococcal antibodies and immunosuppression compared with only 13 patients with low antipneumococcal titres and no immunosuppression $(p=0.008)$. Patients with an IgG papaprotein $(n=63)$ showed significantly lower antipneumococcal IgG antibody titres compared with patients with IgA or light chain only paraproteins $(p=0 \cdot 0058)$.

\section{IMMUNISATION RESPONSES}

Overall response to immunisation was poor; $38 \%$ patients failed to produce any IgG response to Pneumovax II and $35 \%$ and $60 \%$ to tetanus and diphtheria toxoids, respectively (table 4). There was an association between a poor immunisation response to Pneumovax II and a septicaemic episode recorded since diagnosis (retrospective infections included); five of 22 patients with poor or intermediate immunisation responses had had septicaemia compared with none of the 18 good responders

Table 4 Immunisation responses (IgG specific antibodies) in 41 immunised patients

\begin{tabular}{lccccc}
\hline & \multicolumn{2}{l}{$\begin{array}{l}\text { No serious infections } \\
(n=17)\end{array}$} & & \multicolumn{2}{l}{$\begin{array}{l}\text { One or more serious infections } \\
(n=24)\end{array}$} \\
\cline { 2 - 3 } \cline { 5 - 6 } Antigen & Good & Poor** & & Good & Poor $^{* *}$ \\
\hline Pneumovax II $(\mathrm{n}=40)$ & 9 & 8 & & 9 & 14 \\
Tetanus $(\mathrm{n}=40)$ & 10 & 7 & & 12 & 11 \\
Diphtheria $(\mathrm{n}=40)$ & 6 & 11 & & 8 & 15 \\
\hline
\end{tabular}

* Includes retrospectively defined infections (with good documentary evidence).

** Includes poor and intermediate immunisation responses.

One patient missed immunisation with Pneumovax and another with diphtheria/tetanus.

Table 5 IgG subclass levels ( $g / l)$ (results represented as median (range))

\begin{tabular}{lllll}
\hline Patient group & $\operatorname{Ig} G 1(g / l)$ & $\operatorname{Ig} G 2(g / l)$ & $\operatorname{IgG3}(g / l)$ & $\operatorname{IgG4}(g / l)$ \\
\hline Laboratory normal range & $4 \cdot 22-12 \cdot 92$ & $1 \cdot 17-7 \cdot 47$ & $041-1 \cdot 29$ & $0-2 \cdot 91$ \\
Controls $(\mathrm{n}=24)$ & $5 \cdot 20$ & 3.59 & 0.80 & $0.42(0-1 \cdot 22)$ \\
& $(2 \cdot 08-9 \cdot 58)$ & $(1 \cdot 45-5 \cdot 84)$ & $(0 \cdot 33-1 \cdot 42)$ & \\
Myeloma & & & & \\
all $(\mathrm{n}=41)$ & $3 \cdot 00(0-6 \cdot 00)$ & $0.52(0-3 \cdot 7)$ & $0.19(0-1 \cdot 2)$ & $0.05(0-0.9)$ \\
serious infections $\left(\mathrm{n}=24^{*}\right)$ & $2 \cdot 20$ & 0.52 & 0.19 & 0.04 \\
no serious infections $(\mathrm{n}=13)$ & $3 \cdot 10$ & 1.30 & 0.40 & 0.12 \\
\hline
\end{tabular}

Includes only non-paraprotein IgG subclass levels (where the paraprotein was IgG, measurement of the paraprotein subclass was excluded).

* Includes well documented retrospective infections in nine patients. $(p=0.03)$, although there were no such associations for other infections including pneumonia, upper respiratory tract infection or herpes zoster infection. Poor response to Pneumovax II was not significantly associated with a poor response to tetanus or diphtheria toxoids nor with disease stage or performance status at either diagnosis or study entry.

\section{IGG SUBCLASS LEVELS}

IgG subclass levels were measured in the 41 immunised patients and were universally reduced compared with a subgroup of the control population $(n=24)$. IgG2 concentrations were significantly inversely correlated with postimmunisation antipneumococcal antibody levels $(p<0.001)$. There were no similar associations for antidiphtheria or antitetanus titres or with other IgG subclasses. Low IgG2 and low IgA levels also showed a significant correlation in those patients in whom the paraprotein was IgG1, IgG3 and light chain ( $p<0.001 ;$ IgA and IgG2 myelomas excluded). Levels of all non-paraprotein IgG subclasses were significantly lower in patients with IgG paraproteins when compared with those with IgA/light chain only paraproteins $(p=0.005)$. There were no significant differences in IgG subclass measurements between the two groups of patients with and without serious infections (table 5).

INFECTION SEVERITY, SITE AND TYPE

Of the 78 episodes of serious infection which occurred during the study, there were 33 episodes of major infection (including 11 infections at the time of death) and 45 episodes of moderate infection. Ninety four episodes of minor infection were recorded in 48 patients during the same period. There were 31 deaths during the study period; infection was considered to be a contributory factor in 11 . Most infections originated in the respiratory tract; 14 of 22 (64\%) major infections, 19 of 45 $(42 \%)$ moderate infections and six of the 11 $(55 \%)$ infections at the time of death (table 2). Twenty two patients suffered two or more serious infections (four patients had three or more serious infections) but infections were not confined to a single site nor was there incomplete resolution of individual infections to suggest relapsing infection.

Nineteen of 22 major infections, 31 of 45 moderate infections and all of the 11 infections at the time of death were classified as bacterial in origin, although positive bacterial cultures were obtained in only 10 major, 10 moderate and five infective deaths. The predominant 
Table 6 Patient and disease characteristics in relation to serious infection

\begin{tabular}{|c|c|c|c|}
\hline Characteristics examined & No serious infections & Serious infections & $p$ value \\
\hline Number of patients & 53 & 49 & \\
\hline $\begin{array}{l}\text { Sex } \\
\text { male } \\
\text { female }\end{array}$ & $\begin{array}{l}22 \\
31\end{array}$ & $\begin{array}{l}33 \\
16\end{array}$ & 0.01 \\
\hline $\begin{array}{l}\text { Age } \\
\qquad 70 \text { years } \\
>70 \text { years }\end{array}$ & $\begin{array}{l}34 \\
19\end{array}$ & $\begin{array}{l}28 \\
21\end{array}$ & NS \\
\hline $\begin{array}{l}\text { Disease stage } \\
\text { I } \\
\text { II } \\
\text { III }\end{array}$ & $\begin{array}{r}8 \\
17 \\
28\end{array}$ & $\begin{array}{r}4 \\
11 \\
34\end{array}$ & NS \\
\hline $\begin{array}{l}\text { Performance status } \\
\text { asymptomatic } \\
\text { minimal symptoms } \\
\text { restricted activity } \\
\text { bedbound }\end{array}$ & $\begin{array}{r}28 \\
24 \\
1 \\
0\end{array}$ & $\begin{array}{r}13 \\
27 \\
6 \\
3\end{array}$ & 0007 \\
\hline $\begin{array}{l}\text { Chemotherapy (since diagnosis*) } \\
\text { none } \\
\text { oral alkylating agent } \\
\text { combination regimes } \\
\text { intensive** }\end{array}$ & $\begin{array}{l}\left(\mathrm{n}=36^{*}\right) \\
1 \\
22 \\
13 \\
2\end{array}$ & $\begin{array}{l}\left(\mathrm{n}=66^{*}\right) \\
2 \\
40 \\
24 \\
10\end{array}$ & NS \\
\hline $\begin{array}{l}\text { Immunosuppression: mean (median } \\
\text { serum IgG (range } 1 \cdot 2-10 \cdot 2 \text { ) } \\
\text { serum IgA (range } 0-2 \cdot 6 \text { ) } \\
\text { serum IgM (range } 0-3 \cdot 1 \text { ) }\end{array}$ & $\begin{array}{l}\text { vel }(\mathrm{g} / \mathrm{l}) \text { at study entry } \\
6 \cdot 79(6 \cdot 1) \\
1 \cdot 20(0 \cdot 40) \\
0 \cdot 46(0 \cdot 30)\end{array}$ & $\begin{array}{l}3.78(4.11) \\
0.36(0.30) \\
0.45(0.30)\end{array}$ & $\begin{array}{l}<0.0001 \\
<0.0001 \\
\text { NS }\end{array}$ \\
\hline
\end{tabular}

** All had previously received alkylating agents or combination regimes.

organisms isolated were Streptococcus pneumoniae (10/11 Gram positive isolates) and $E$ coli (nine of 14 Gram negative isolates). The other infections included known viral infections (six due to herpes zoster virus) and five which were classified as "pyrexia of unknown origin". Infections at the time of death were excluded from further analysis where stated as all patients had end stage disease at the time of death and the relative contributions of infection and myeloma to death were impossible to assess.

\section{TIMING OF INFECTION}

The infection rate during induction chemotherapy was 1.58 serious infections per patient year (21 infections in 13.3 years of observation) compared with 0.49 in plateau phase (27 infections during 55.7 years) and 1.90 during relapsed/progressive disease phase (30 infections during $15 \cdot 8$ years). The rates during both induction and relapsed/progressive disease were significantly higher than the infection rate in plateau phase (both $\mathrm{p}<0.001$ ). Forty five $(58 \%)$ patients had received specific myeloma chemotherapy within 28 days before the onset of a serious infection, whereas $33(42 \%)$ had not received any such treatment before sustaining a serious infection. Only two patients demonstrated absolute neutrophil counts of less than $1000 \times 10^{6} / 1$ in relation to serious infection so further analysis was not possible. There was no significant seasonal variation in the number of serious infections occurring in any three month period.

PREDICTORS OF INFECTION

Patient and disease characteristics were examined in the two groups of patients with and without serious infections (infections in association with death were excluded). Nonparaprotein serum IgG and IgA levels at entry were both significantly reduced in patients with compared with those without serious infections $(p<0.001)$. No such differences were demonstrated for serum IgM levels. There were more men than expected in the group with serious infection; the serious infection rate in men was 1.00 infections per patient year, twice the rate of 0.53 in women $(p=0.02)$. In an attempt to account for these differences, smoking habits were analysed and showed that significantly fewer men than women had never smoked (18 men compared with 29 women: $p=0.003)$. There were no significant differences between the serious infection groups for the other parameters examined (table 6).

\section{Discussion}

Previous studies of infection rates in myeloma have suggested an association between infection and specific myeloma chemotherapy. Savage $e t a l^{2}$ found that 57 of 64 infective episodes in patients with advanced disease occurred after chemotherapy but there was no temporal association between neutropenia and infection. Active disease, as reflected by paraprotein level, was a risk factor. Our study was primarily concerned with patients with relatively stable, predominantly plateau phase disease receiving low dose or no chemotherapy (table 6) permitting the influence of other infection risk factors such as immunological deficiencies to be examined. We confirmed that active disease in patients undergoing chemotherapy was significantly associated with serious infection.

The possible contribution of polyclonal humoral immunosuppression to infection in myeloma was examined in detail because of its contribution to susceptibility to infection in other lymphoproliferative disorders ${ }^{13}$ and the likelihood that it contributes to bacterial infections of the types described in myeloma. ${ }^{35}$ 
The majority of patients $(75 \%)$ demonstrated immunosuppression of at least one non-paraprotein immunoglobulin class. When immunosuppression was broken down by immunoglobulin class, non-paraprotein serum IgG and IgA levels were significantly lower in patients who sustained serious infections. Serum IgM levels failed to show this difference. No correlation between humoral immunosuppression and tumour load (as assessed by clinical staging) could be identified, a similar finding to earlier studies. ${ }^{7}$

Baseline specific antibody titres and IgG subclass levels have been suggested to be more sensitive markers of immunosuppression in primary immune deficiency ${ }^{14}$ and in myeloma. ${ }^{15}$ We demonstrated noticeably reduced specific antibody titres and non-paraprotein IgG subclass levels in patients with myeloma compared with a healthy control population of a similar age. The correlation between increased infection risk and low antipneumococcal and low anti- $E$ coli LPS titres confirms the findings of Stoll et al. ${ }^{15} \mathrm{IgG}$ antibody responses to immunisation were poor in over half the patients when challenged with either tetanus or diphtheria toxoids (both protein antigens) or polysaccharide pneumococcal capsular antigens as shown previously, ${ }^{16}$ although this had not been related to infection in the past. The significant association between poor antibody responses to pneumococcal antigens and an increased risk of septicaemia $(p=0.03)$ and a similar, but not significant, trend for other serious infections suggest that patients at particular risk of serious infection can be identified. Furthermore, the significant association between low IgG2 levels and poor pneumococcal IgG antibody responses on the one hand and low serum IgA levels on the other indicate that selective immunosuppression, particularly of IgG responses to carbohydrate antigens, may account for this increased susceptibility to bacterial infections.

Infection clustering at the time of initial myeloma diagnosis has been suggested which may be because of a number of factors including high tumour load, anaemia, compromised renal function, hypercalcaemia, initial chemotherapy, or a combination of these factors. ${ }^{24}$ These factors are also likely to be interacting at times of disease relapse. We examined timing of infection by investigating the phase of the disease in which serious infection occurred. The rate was highest during initial induction chemotherapy or during progressive disease, when it was increased fourfold compared with the rate during plateau phase. Nevertheless, a reasonable proportion of infections still occurred in plateau phase.

Factors other than humoral immunity were studied in relation to infection risk. Chemotherapy regimes have been suggested as important but almost half of the patients who suffered serious infections had not received chemotherapy within 28 days before their infection. Furthermore, there was no significant increase in the proportion of patients receiving aggressive chemotherapy who sustained serious infections compared with those with no serious infections. Likewise neutropenia (less than $1000 \times 10^{6}$ neutrophils per litre) was seen only twice in association with serious infection and patients who suffered serious infections did not have significantly lower neutrophil counts compared with patients who had no serious infections. The lack of any significant direct correlation between chemotherapy, neutrophil counts and infection is not surprising in this group of patients with disease in plateau phase.

Previous studies of infection in myeloma have been retrospective and relied heavily on positive microbiological culture. Our data showed an overall rate of 0.92 serious infections per patient year which is consistent with earlier data (reported rates between 0.8 and 2.22 infections per patient year ${ }^{1-4}$ ) and considerably higher than the rate in age matched patients in hospital for other reasons. ${ }^{1}$ Breakdown of infection by site showed a predominance of serious infections affecting the respiratory tract, followed by urinary tract infection, septicaemia, and other pyrexias of unkown origin, ${ }^{13}$ similar to earlier studies. Bacterial skin infections were not observed frequently, although localised herpes zoster was more frequent than in earlier studies perhaps because of the high number of long term survivors included in this study. The low rate of positive bacterial culture precluded any comment on changing patterns of infecting organisms; isolates were predominantly $S$ pneumoniae and $E$ coli, the organisms chosen for measurement of specific antibody titres. There was no seasonal variation in infection despite the high proportion of respiratory tract infections.

Examination of patient characteristics revealed only male sex to be associated with a significantly increased risk of infection. This is in accordance with the finding of shorter disease survival in men; ${ }^{17}$ an increased infection rate as the cause for shortened survival has not been investigated. Analysis of smoking habits proved unhelpful in predicting infection risk despite the predominance of respiratory tract infections although it may have explained, at least in part, the increased risk of infection in men. Disease characteristics were also unhelpful in predicting infection; neither paraprotein type, disease stage nor presence of urinary free light chains proved to be significant predictive factors. Performance status did show an association, with the more symptomatic patients sustaining more serious infections, confirming the findings of the disease phase analysis. Creatinine and haemoglobin concentrations were examined in isolation to investigate their individual contributions as distinct from their combined effect on disease staging, but no significant associations were identified.

In conclusion, this study, largely confined to patients with plateau phase myeloma, shows a continued increased risk of bacterial infection, independent of chemotherapy and associated with poor IgG antibody production. Those at greatest risk can be identified by their poor IgG response to Pneumovax II immunisation in addition to low levels of naturally occurring antibodies to pneumococcal capsular polysaccharides and $E$ coli LPS. This group of 
patients might be expected to benefit from regular, prophylactic replacement intravenous immunoglobulin containing the deficient antibodies to reduce their risk of infection. A double blind, placebo controlled study investigating this aspect of infection in myeloma has recently been reported and has shown a significant protective effect. ${ }^{18}$

This research was supported by an educational grant from Baxter Healthcare Corporation, Hyland Division, Glendale, California, USA. We thank Mrs Beckley, Mrs Henley and Mrs Welby for typing the manuscript and Drs Emerson, Wainscoat Littlewood, and Ardeman for permission to include their patients. Dr R Mayon-White kindly read the manuscript.

1 Twomey JJ, Houston MB. Infections complicating multiple myeloma and chronic lymphocytic leukaemia. Arch Intern Med 1973;132:562-5.

2 Savage DG, Lindenbaum J, Garrett TJ. Biphasic pattern of bacterial infection in multiple myeloma. Ann Intern Med 1982:96:47-50.

3 Fahey JL, Scoggins R, Utz JP, Szwed CF. Infection, antibody response and gamma globulin components in multiple response and gamma globulin components in multiple
myeloma and macroglobulinaemia. Am $₹$ Med 1963;35: myeloma

4 Perri RT, Hebbel R, Oken MM. Influence of treatment and response status on infection risk in multiple myeloma. $\mathrm{Am}$ $7 \mathrm{Med}$

5 Meyers RB. Hirschman SZ, Axelrod JA. Current patterns of infection in multiple myeloma. Am $\mathcal{f}$ Med 1972;52 87-92.

6 Kyle RA. Multiple Myeloma. An update on diagnosis and management. Acta Oncologica 1990;29:1-8.
7 Medical Research Council Working Party Report on the first myelomatosis trial. Br $\mathcal{f}$ Haematol 1973;24:123-39. 8 Windebank KP, Faux JA, Chapel HM. ELISA determination of IgG antibodies to pneumococcal capsular polysaccharides in a group of children. $\mathcal{f}$ Immunol Methods 1987;104:143-8

9 Cuzick J, DeStavola BL, Cooper EH, Chapman C, MacLennan ICM. Long term prognostic value of serum beta 2 microglobulin in myelomatosis. $\mathrm{Br} \mathcal{F}$ Haematol 1990;75: 506-10.

10 Durie BG, Salmon SE. A clinical staging system for multiple myeloma. Cancer 1975;36:842-54.

11 Medical Research Council. Myelomatosis: comparison of melphalan and cyclophosphamide therapy. BMF 1971;i: melphat.

12 Rosner B. In: Fundamentals of biostatics. 3rd edn. Boston: PWS-Kent Publishing Co, 1990:432-6.

13 Griffiths H, Lea J, Bunch C, Lee M, Chapel H. Predictors of infection in chronic lymphocytic leukaemia (CLL). Clin Exp Immunol 1992;89:374-7.

14 Cunningham Rundles C. Clinical and immunologic analyses of 103 patients with common variable immunodeficiency. 7 Clin Immunol 1989;91:22-33.

15 Stoll C, et al. Serum antibodies against common antigens of bacterial lipopolysaccharide in healthy adults and patients with multiple myeloma. Infection 1985;13 (part 3):115-19.

16 Birgens HS, Espersen F, Hertz JB, Pedersen FK, Drivsholm A. Antibody response to pneumococcal vaccination in A. Antibody response to pneumococcal vaccination in 30 .

17 Cherng NC, Asal NR, Kuebler JP, Lee E, Solanki D. Prognostic factors in multiple myeloma. Cancer 1991;67: 3150-6.

18 Chapel HM, Lee M, Hargreaves R, Pamphilon D, Prentice AG. Randomised trial of intravenous immunoglobulin as prophylaxis against infection in plateau-phase multiple myeloma. The UK Group for Immunoglobulin Replacement Therapy in Multiple Myeloma. Lancet 1994; 343:1059-63. 